\title{
Caracterización y selección de sitios de excavación del carpintero lomo blanco (Campephilus leucopogon) en dos regiones forestales del noroeste Argentino
}

\author{
Characterization and selection of excavation sites by Campephilus leucopogon \\ in two forest regions in Northwestern Argentina
}

\author{
Sebastian Albanesi a, Alejandro Schaaf ${ }^{\text {a,b }}$, Constanza Vivanco ${ }^{\text {a,b }}$, Luis Rivera ${ }^{\text {a,b }}$, Natalia Politi ${ }^{\text {a,b,* }}$ \\ ${ }^{a}$ Fundación CEBio, San Salvador de Jujuy, Argentina. \\ *Autor de correspondencia: ${ }^{b}$ Universidad Nacional de Jujuy, Centro de Investigaciones y Transferencia de Jujuy, \\ CONICET, San Salvador de Jujuy, Argentina, npoliti@conicet.gov.ar
}

\begin{abstract}
SUMMARY
Woodpeckers are considered engineering species of forests and are sensitive to changes in forests composition and structure. Northwestern Argentina contains the largest area of native forest of the country. The objectives of this study are to characterize sites where the Cream-backed woodpecker (Campephilus leucopogon) excavates its nesting or roosting cavities and identify selection patterns of those sites in the Piedmont forest of the Southern Yungas and Dry Chaco forest of Northwestern Argentina. In both forest regions transects were conducted to search for excavated cavities by the Cream-backed woodpecker. Trees with and without cavities and random plots where characterized. Both in the Piedmont and Chaco forest, most of the excavated cavities found were in live trees of more than $60 \mathrm{~cm}$ of diameter at breast height. Excavated cavity height was greater in the Piedmont than in the Chaco forest. The Cream-backed woodpecker selected for excavation Calycophyllum multiflorum and Amburana cearensis in the Piedmont forest and Aspidosperma quebracho-blanco and Chorisia insignis in the Chaco forest. The results obtained in this study allow proposing guidelines on how to improve forest management by retaining key structural attributes for the Cream-backed woodpecker. The species maintenance in managed forest will determine the amount of cavities available for many other non-excavator species.
\end{abstract}

Key words: logging, biodiversity, cavity, woodpecker, selection.

\section{RESUMEN}

Los pájaros carpinteros (Aves, Picidae) son considerados especies ingenieras de ecosistemas, y son sensibles a cambios en la composición y estructura de los bosques. El noroeste Argentino contiene la mayor superficie de bosque nativo del país. El objetivo de este trabajo fue caracterizar los sitios donde el carpintero lomo blanco (Campephilus leucopogon) excava huecos e identificar patrones de selección de sustratos de excavación en la selva pedemontana de las Yungas Australes y en el bosque de dos quebrachos del Chaco Seco del noroeste Argentino. En las dos regiones forestales se recorrieron transectas en busca de huecos excavados por el carpintero lomo blanco. Se caracterizaron los árboles con huecos y se compararon con árboles sin huecos evaluados en parcelas ubicadas al azar. En la selva pedemontana y bosque chaqueño, la mayoría de los huecos excavados por el carpintero lomo blanco se encontraron en árboles vivos con grandes diámetros a la altura del pecho $(>60 \mathrm{~cm})$, significativamente mayores que los de los árboles disponibles. La altura de los huecos excavados fue mayor en la selva pedemontana que en el bosque chaqueño, en concordancia con la mayor altura promedio de la primera. El carpintero lomo blanco seleccionó para excavar huecos ejemplares de Calycophyllum multiflorum y Amburana cearensis en la selva pedemontana y de Aspidosperma quebracho-blanco y Chorisia insignis en el bosque chaqueño. Estos resultados permiten proponer recomendaciones de manejo forestal para retener atributos estructurales clave para el carpintero lomo blanco.

Palabras clave: aprovechamiento forestal, biodiversidad, huecos, pájaro carpintero, selección.

\section{INTRODUCCIÓN}

Los pájaros carpinteros (Aves, Picidae) son considerados especies clave e ingenieras de ecosistemas en los bosques, ya que crean, modifican y mantienen hábitats esenciales para otros organismos (Cornelius et al. 2008). Los pájaros carpinteros excavan huecos en árboles como sitios de nidificación y dormidero, por lo que se los denomina usuarios primarios de huecos (Cornelius et al. 2008).
Debido a que los pájaros carpinteros tienen requerimientos de hábitat altamente específicos, son un grupo sensible a los cambios en la composición y estructura en los bosques (Cornelius et al. 2008). En muchas regiones boscosas del mundo, la falta de planificación ha hecho que muchas poblaciones de pájaros carpinteros hayan disminuido o hayan sido local o regionalmente extirpadas debido a prácticas forestales inadecuadas (Aitken y Martin 2007). Se ha sugerido que la disponibilidad de árboles adecuados para ex- 
cavar limita la densidad de muchas poblaciones de pájaros carpinteros (Cornelius et al. 2008). Dado que los pájaros carpinteros tienen un rol fundamental en generar huecos que luego son usados por otras especies, la disminución o extirpación de este grupo de aves puede tener un efecto directo sobre muchas especies que usan huecos (Aitken y Martin 2007).

Existen aproximadamente 255 especies de pájaros carpinteros que se distribuyen por gran parte del mundo, exceptuando Australia, Nueva Zelanda y Madagascar (Winkler y Christie 2002). En Argentina, se han registrado 28 especies de pájaros carpinteros de las cuales cuatro pertenecen al género Campephilus (C. leucopogon, C. maguellanicus, C. melanoleucos y C. robustus) (Winkler y Christie 2002). Los carpinteros del género Campephilus son de gran tamaño (entre 35 y $44 \mathrm{~cm}$ ) y se encuentran desde las selvas del norte de Argentina hasta los bosques patagónicos (Winkler y Christie 2002). El carpintero lomo blanco (C. leucopogon (Valenciennes, 1826)) se distribuye en el norte y centro de Argentina, norte y centro de Bolivia, oeste y centro de Paraguay, norte de Uruguay y sudeste de Brasil, habitando desde bosques húmedos de montaña hasta bosques xerófilos y sabanas, y un amplio gradiente altitudinal (400-2500 m s.n.m.) (Winkler y Christie 2002). En Argentina, se han registrado unos pocos nidos de esta especie, entre julio y diciembre, en huecos en ramas y troncos de árboles, con un diámetro de entrada del hueco de $11 \mathrm{~cm}$ y una profundidad interna del hueco de $38 \mathrm{~cm}$ (de la Peña 2005). Se menciona además, que en Brasil se registró un nido de carpintero lomo blanco en julio con un pichón y que los huecos están entre 6 y $8 \mathrm{~m}$ de altura en árboles o palmeras (Winkler y Christie 2002). Los huecos excavados por el carpintero lomo blanco son utilizados por varias especies de aves que nidifican en huecos de árboles (e.g., Amazona tucumana, Pionus maximiliani, Falco rufigularis) (Politi et al. 2010).

El noroeste argentino contiene la mayor superficie de bosque nativo del país y a su vez presenta la mayor tasa de transformación de bosques a otros usos de la tierra como áreas urbanas, agrícolas y ganaderas. Los bosques remanentes son aprovechados sin planificación o criterios de sustentabilidad que aseguren la conservación a largo plazo de la biodiversidad. Es necesario entender los requerimientos de hábitat del carpintero lomo blanco para delinear pautas de manejo forestal que aseguren la conservación de esta especie y de otras que dependen de huecos excavados. El objetivo de este trabajo es caracterizar los sitios donde el carpintero lomo blanco excava huecos (nidos y/o dormideros), e identificar los patrones de selección para sitios de excavación en dos tipos de bosques contiguos del noroeste Argentino: la selva pedemontana (ubicado en el piso altitudinal más bajo dentro de la ecorregión de las Yungas Australes) y el bosque de dos quebrachos (perteneciente a la ecorregión del Chaco Seco) en función de la disponibilidad de las especies arbóreas y de las distintas características de los árboles. Si el carpintero lomo blanco usa especies arbóreas más frecuentemente de lo esperado en función de la disponibilidad y si existen diferencias en las características de los árboles usados para excavar comparado con los de árboles no usados, habrá evidencia de una selección de los sitos de excavación.

\section{MÉTODOS}

Área de estudio. El estudio se realizó en dos tipos de bosque del este de la provincia de Jujuy: un sitio con selva pedemontana de la ecorregión de Yungas Australes ubicado en el departamento General Libertador San Martín (23 56,746 S, 6454,389 O) y un sitio con bosque de dos quebrachos de la ecorregión del Chaco Seco ubicado en el departamento de Santa Bárbara (23 39,001 ' S, 64 ${ }^{\circ} 16,163$ ' O). Ambas ecorregiones son contiguas y los sitios se encuentran a una distancia aproximada de $100 \mathrm{~km}$ (figura 1). La selva pedemontana se ubica entre los 400 y $700 \mathrm{~m}$ s.n.m., con una temperatura media anual entre $18-20^{\circ} \mathrm{C}$ y una precipitación media anual de $800 \mathrm{~mm}$, concentrada en los meses de noviembre a marzo. Las especies arbóreas más representativas de la selva pedemontana son: palo blanco (Calycophyllum multiflorum Griseb. (Castelo)), palo amarillo (Phyllostylon rhamnoides Poiss.), lapacho rosado (Tabebuia impetiginosa Mart. ex DC), cedro orán (Cedrela balansae Moç. et Sessé ex DC), roble criollo (Amburana cearensis (Allemâo) A. C. Smith) y urundel (Astronium urundeuva (Allemâo) Engl.). Actualmente, el $90 \%$ de la selva pedemontana ya ha sido transformada y los remanentes se encuentran bajo un aprovechamiento forestal intensivo $\left(5-10 \mathrm{~m}^{3} \mathrm{ha}^{-1}\right)$ y menos de $1 \%$ se encuentra protegido (Brown et al. 2009).

El bosque chaqueño de dos quebrachos cubre llanuras de poca elevación ( $<$ de 400 m s.n.m.) con clima continental cálido y una precipitación media anual de 500 a $800 \mathrm{~mm}$ y una temperatura media anual de $20-23{ }^{\circ} \mathrm{C}$. Entre los árboles más representativos se encuentra el quebracho blanco (Aspidosperma quebracho-blanco Schltdl.), quebracho colorado (Schinopsis lorentzii (Griseb.) Engl.), mistol (Zizipus mistol Griseb.) y yuchán (Chorisia insignis HBK.). El bosque chaqueño de dos quebrachos tiene unas de las tasas absolutas $(0,82 \%$ anual) más elevadas de transformación a nivel mundial de área de bosque a zonas agrícolas debido principalmente a la reciente expansión del cultivo de soja (Glycine max (L.) Merr.) (Gasparri y Grau 2009). Además, posee una intensa extracción de quebrachos (en 1993 en Argentina se reportó la extracción de 822.770 toneladas) para la obtención de madera, taninos y carbón en los bosques remanentes (Gasparri y Grau 2009).

Metodología. Entre los meses de julio del 2009 y octubre del 2010 se recorrieron en la selva pedemontana 10 transectas de $1,5 \mathrm{~km}$ (total $15 \mathrm{~km}$ ) y en el bosque chaqueño 12 transectas de entre 3 y $12 \mathrm{~km}$ (total $50 \mathrm{~km}$ ) siguiendo caminos principales, secundarios, picadas de prospección petrolera y sendas, en busca de huecos excavados por el 


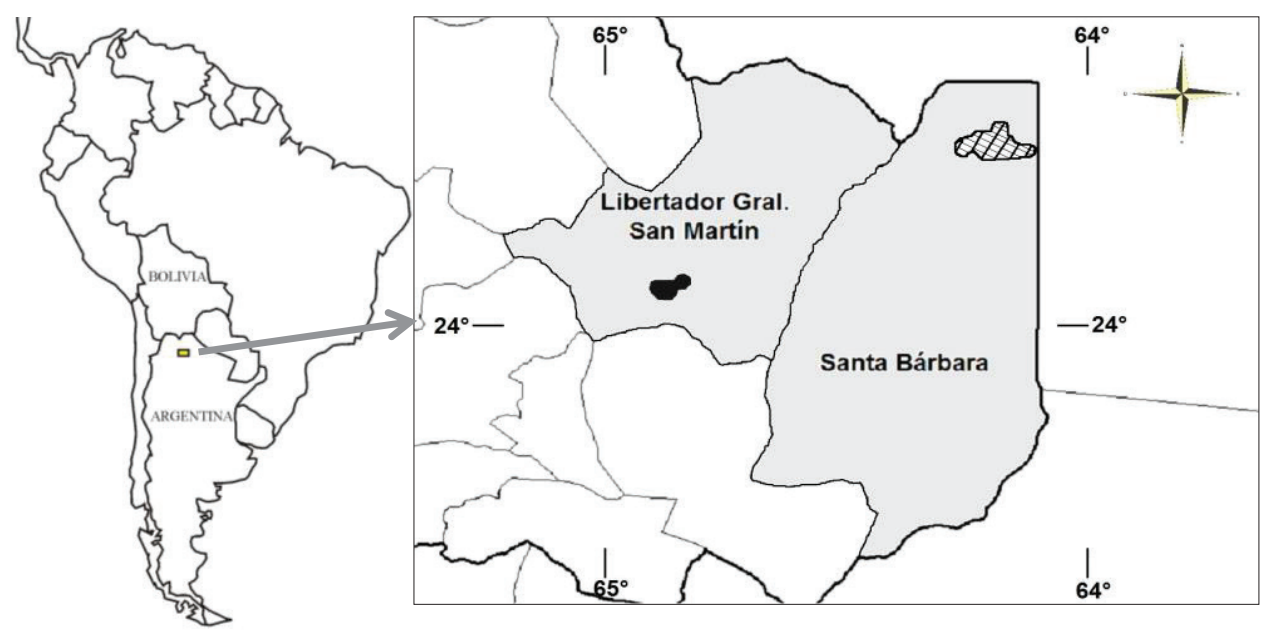

Figura 1. Ubicación del área de estudio (recuadro) en la provincia de Jujuy, Argentina y de los sitios en el departamento Libertador General San Martín (selva pedemontana de las Yungas Australes, área negra) y en el departamento Santa Bárbara (bosque de dos quebrachos del Chaco Seco, área achurada).

Location of the study area in Jujuy Province, Argentina (left) and the study sites (right) in the Department of Libertador General San Martin (Piedmont forest of the Southern Yungas, black area) and Department of Santa Bárbara (Dry Chaco forest, hatched area).

carpintero lomo blanco. En la selva pedemontana, el área aproximada que se recorrió fue de $10 \mathrm{~km}^{2}$ de superficie, y en el bosque chaqueño de dos quebrachos, de $30 \mathrm{~km}^{2}$. Posiblemente, el número de territorios de carpintero lomo blanco en cada una de las áreas estudiadas no supere los 10 , en función de lo que se conoce para otras especies del género y de las observaciones realizadas en los sitios de estudio (Husak y Husak 2003, Politi et al. 2012). Los huecos excavados por el carpintero lomo blanco son fáciles de identificar por la entrada del hueco en forma de gota y el mayor tamaño que los huecos excavados por otros pájaros carpinteros que se encuentran en la región (e.g., carpintero real común (Colaptes melanolaimus), carpintero verdiamarillo (Piculus chrysochloros), carpintero blanco (Melanerpes candidus)). En muestreos de aves en el área de estudio no se registró al carpintero negro (Dryocopus schul$z i)$ que podría excavar huecos de dimensiones similares a las del carpintero lomo blanco. Los huecos del carpintero lomo blanco que se detectaron fueron georeferenciados, se identificó la especie de árbol que albergaba el hueco, y se midieron el diámetro del árbol a la altura del pecho (DAP), la altura del hueco desde el suelo (con un palo extensible de $15 \mathrm{~m}$ ), la altura del árbol (estimada visualmente), y la orientación cardinal de la entrada del hueco, con brújula. Se determinó también la ubicación del hueco en el árbol (i.e., tronco, rama primaria, secundaria, terciaria o cuaternaria) y el sustrato en el árbol que contiene el hueco (i.e., vivo o muerto). Para caracterizar el bosque en la selva pedemontana, se demarcaron al azar 20 parcelas circulares de 0,05 ha (total 1 ha) y en el bosque chaqueño de dos quebrachos, 14 parcelas rectangulares de 0,1 ha (total 1,5 ha). Para todos los árboles $>10 \mathrm{~cm}$ DAP en cada parcela, se estimó la altura del árbol, se midió el DAP, se registró la especie y se inspeccionaron para determinar si tenían huecos del carpintero lomo blanco. Los árboles muertos en pie dentro de estas parcelas se registraron cualitativamente como una categoría adicional a las especies de los árboles vivos.

Análisis de los datos. Se calculó una densidad aproximada de huecos excavados por el carpintero lomo blanco como el número de huecos encontrados por el área recorrida en cada tipo de bosque. Se realizaron comparaciones de las variables de las parcelas de vegetación y de las características del árbol con hueco entre los dos tipos de bosque y de la selección de los sitios de excavación dentro de cada tipo de bosque. Todas las variables se probaron para evaluar la normalidad de su distribución, pero dado que no cumplieron dicho supuesto de la estadística paramétrica, se utilizaron pruebas no paramétricas. Se calcularon el área basal, la densidad, el DAP y la altura promedio de los árboles en las parcelas de vegetación, y se determinaron las especies arbóreas dominantes. Se compararon los sitios a base de las variables cuantitativas de la vegetación previamente calculadas con la prueba no paramétrica U de Mann Whitney. Los árboles utilizados para excavación se compararon entre ambos sitios, en base al DAP y a la altura de los huecos, utilizando la prueba no paramétrica U de Mann Whitney.

Dentro de cada tipo de bosque, se comparó el DAP de los árboles utilizados por el carpintero para excavar huecos con el de las parcelas de vegetación (considerando solo los árboles sin huecos de las parcelas) con la prueba no paramétrica U de Mann Whitney. Se evaluó la selección del carpintero lomo blanco para excavar huecos en las distin- 
tas especies arbóreas a través del índice de selección estandarizado de Manly (Manly et al. 1993). Para cada especie arbórea se comparó el número total de árboles (disponibilidad) con el número de árboles con huecos excavados (uso). El número total de árboles se determinó considerando solo aquellos árboles con tamaño igual o mayor al DAP mínimo con hueco excavado por el carpintero lomo blanco encontrado en este trabajo. Se calcularon los intervalos de confianza de Bonferroni para cada índice de selección y si los intervalos de confianza de cada especie arbórea no se superponían, se evaluó el nivel de significancia. Para determinar si existen diferencias respecto a la ubicación del hueco en el árbol (tronco, rama primaria, secundaria, terciaria o cuaternaria) y al tipo de sustrato (vivo o muerto) usado por el carpintero lomo blanco para excavar huecos se realizaron pruebas $\chi^{2}$ para diferencias de proporciones. Para todos los análisis se utilizó el programa INFOSTAT y un nivel de significancia de $P<0,05$. Se evaluó si existía un patrón en la orientación de las entradas de los huecos excavados en cada uno de los tipos de bosques, a través de la prueba de uniformidad de Rayleigh (Fisher 1993). Para este análisis se utilizó el programa Oriana con un nivel de significancia de $P<0,05$.

\section{RESULTADOS}

En la selva pedemontana se encontró un total de 41 huecos excavados (con una densidad aproximada de 0,041 huecos excavados ha-1) de carpintero lomo blanco en ocho especies de árboles y en el bosque chaqueño de dos quebrachos un total de 45 huecos excavados $(0,015$ huecos excavados ha-1 ${ }^{-1}$ en cinco especies de árboles (cuadro 1). En la selva pedemontana se identificaron 65 especies de árboles, mientras que en el bosque chaqueño de dos quebrachos se identificaron 19 especies de árboles.

Comparación entre tipos de bosque. En la selva pedemontana se encontró significativamente mayor densidad de árboles $\left(418 \pm 133,4\right.$ vs. $310 \pm 112,3$ individuos ha ${ }^{-1}$, respectivamente; $P<0,01)$, área basal $\left(25,40 \pm 9.20 \mathrm{~m}^{2} \mathrm{ha}^{-1}\right.$ vs. $\left.20,95 \pm 6,45 \mathrm{~m}^{2} \mathrm{ha}^{-1} ; P<0,01\right)$, DAP $(25,00 \pm 5,00 \mathrm{~cm}$ vs. $18,34 \pm 10,84 \mathrm{~cm} ; P<0,01)$ y altura $(17,43 \pm 5,52 \mathrm{~m}$ vs. $10,59 \pm 4,14 \mathrm{~m} ; P<0,01)$ que en el bosque chaqueño de dos quebrachos. El DAP de los árboles con huecos excavados no difirió significativamente entre la selva pedemontana $(63,21 \pm 12,65 \mathrm{~cm})$ y el bosque chaqueño de dos quebrachos $(58,99 \pm 18,68 \mathrm{~cm} ; P=0,58)$, mientras que la altura de los huecos excavados fue significativamente mayor en la selva pedemontana $(14,91 \pm 5,29 \mathrm{~m})$ que en el bosque chaqueño de dos quebrachos (7,05 $\pm 2,43 \mathrm{~m} ; P<0,01)$.

Comparación dentro de la selva pedemontana. Calycophyllum multiflorum y Amburana cearensis fueron utilizadas más de lo esperado por el carpintero lomo blanco para excavar huecos y Phyllostylon rhamnoides (J. Poiss.) Taub. fue utilizado menos de lo esperado (cuadro 1). El DAP promedio de los árboles con huecos $(63,21 \pm 12,65 \mathrm{~cm})$ fue significativamente mayor al DAP de los árboles sin hue$\cos (28,19 \pm 13.66 \mathrm{~cm} ; \mathrm{W}=21819, P<0,01)$ (cuadro 2). Los huecos excavados se encontraron mayormente en sustratos vivos $(74 \%)$ respecto a sustratos muertos $(26 \%)$ $\left(\chi^{2}=0,46 ; P<0,01\right)$. Se encontró significativamente mayor proporción de huecos excavados en troncos $(37 \%)$ que en ramas $\left(\chi^{2}=0,27 ; P=0,02\right)$ (figura 2). La orientación de los huecos excavados tuvo una distribución uniforme (Rayleigh $\mathrm{Z}=0,18 ; P=0,84$ ).

Comparación dentro del bosque chaqueño. Aspidosperma quebracho-blanco y Chorisia insignis fueron utilizadas más de lo esperado para excavar huecos (cuadro 1). El DAP promedio de los árboles con huecos $(58,99 \pm 18,68 \mathrm{~cm})$ fue significativamente mayor al DAP de los árboles sin huecos $(26,25 \pm 11.04 \mathrm{~cm} ; \mathrm{W}=7125 ; P<0,01)$ (cuadro 2). La mayoría de los huecos excavados se encontraron en sustratos vivos $(89 \%)$ respecto a sustratos muertos (11\%) $\left(\chi^{2}=0,78 ; P<0,01\right)$. No se encontró diferencia significativa entre la proporción de huecos excavados en troncos y en ramas $\left(\chi^{2}=0,11 ; P=0,30\right)$ (figura 2$)$. La orientación de los huecos excavados tuvo una distribución uniforme (Rayleigh $\mathrm{Z}=0,75 ; P=0,47$ ).

\section{DISCUSIÓN}

En la selva pedemontana de las Yungas Australes y en el bosque chaqueño de dos quebrachos, la mayoría de los huecos se encontraron en árboles vivos o en sustratos vivos. Los árboles muertos pueden ser más fáciles para excavar huecos pero el árbol tiene mayor probabilidad de caerse y menor aislamiento térmico que los árboles vivos (Politi et al. 2010). La mayoría de los huecos excavados

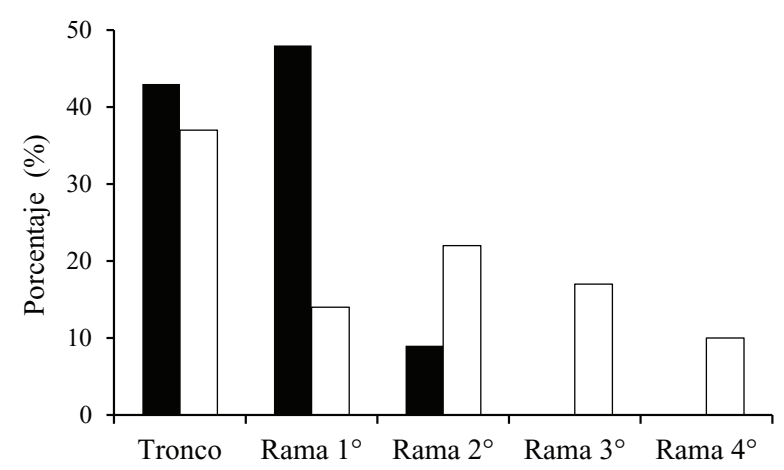

Figura 2. Ubicación en el árbol de los huecos excavados por Campephilus leucopogon en la selva pedemontana de las Yungas Australes (negro) y en el bosque de dos quebrachos del Chaco Seco (blanco) de la provincia de Jujuy, Argentina.

Location in the tree of excavated cavities by Campephilus leucopogon in Piedmont forest of Southern Yungas (black bars) and Dry Chaco forest (white bars) in Jujuy Province, Argentina. 
Cuadro 1. Número $(\mathrm{N})$ y porcentaje (\%) de huecos excavados por Campephilus leucopogon según las especies arbóreas, la disponibilidad (porcentaje de importancia relativa) de esas especies arbóreas en las parcelas de vegetación realizadas y la selección por C. leucopogon de esas especies arbóreas "a favor" (es decir, cuando la proporción observada (Po) de uso de ese recurso es mayor que la proporción esperada (Pe) en función de la disponibilidad; +), "similar" (cuando la Po de uso de ese recurso es igual a la Pe; =), o "menor" (cuando la Po de uso de ese recurso es menor a la Pe; --) en la selva pedemontana de las Yunga Australes y en el bosque de dos quebrachos del Chaco Seco de la provincia de Jujuy, Argentina.

Number (N) and percentage (\%) of cavities excavated by Campephilus leucopogon according to tree species, availability of those tree species (relative importance percentage) in vegetation plots, and positive selection by C. leucopogon of those tree species (i.e., when the observed proportion ( $\mathrm{Po}$ ) of use of that resource is higher than the expected proportion ( $\mathrm{Pe}$ ) based on its availability; + ), no selection (i.e., when Po is equal to Pe; =), and negative selection (i.e., when Po is less than Pe; --) in the Piedmont forest of Southern Yungas and the Dry Chaco forest in Jujuy Province, Argentina.

\begin{tabular}{|c|c|c|c|c|c|c|c|c|c|c|c|c|}
\hline \multirow{3}{*}{$\begin{array}{l}\text { Especie de árbol } \\
\text { Calycophyllum } \\
\text { multiflorum }\end{array}$} & \multicolumn{6}{|c|}{ Selva pedemontana } & \multicolumn{6}{|c|}{ Bosque chaqueño } \\
\hline & \multirow{2}{*}{$\begin{array}{c}\begin{array}{c}\text { Disponibilidad } \\
\text { de árboles } \\
\%\end{array} \\
7\end{array}$} & \multicolumn{2}{|c|}{$\begin{array}{l}\text { Número } \\
\text { de huecos } \\
\text { excavados } \\
(\%)\end{array}$} & \multirow{2}{*}{$\begin{array}{c}\text { Po } \\
0,39\end{array}$} & \multirow{2}{*}{$\begin{array}{c}\mathrm{Pe} \\
0,08\end{array}$} & \multirow{2}{*}{$\begin{array}{c}\text { Selección } \\
+\end{array}$} & \multirow{2}{*}{$\begin{array}{c}\begin{array}{c}\text { Disponibilidad } \\
\text { de árboles } \\
\%\end{array} \\
0\end{array}$} & \multicolumn{2}{|c|}{$\begin{array}{c}\text { Número } \\
\text { de huecos } \\
\text { excavados } \\
(\%)\end{array}$} & \multirow{2}{*}{$\begin{array}{c}\text { Po } \\
-\end{array}$} & \multirow{2}{*}{$\begin{array}{c}\text { Pe } \\
-\end{array}$} & \multirow{2}{*}{$\begin{array}{c}\text { Selección } \\
-\end{array}$} \\
\hline & & 16 & $(39,02)$ & & & & & 0 & - & & & \\
\hline $\begin{array}{l}\text { Amburana } \\
\text { cearensis }\end{array}$ & 1 & 7 & $(17,07)$ & 0,17 & 0,02 & + & 0 & 0 & - & - & - & - \\
\hline $\begin{array}{l}\text { Astronium } \\
\text { urundeuva }\end{array}$ & 6 & 3 & $(7,32)$ & 0,07 & 0,10 & $=$ & 0 & 0 & - & - & - & - \\
\hline $\begin{array}{l}\text { Cedrela } \\
\text { balansae }\end{array}$ & 1 & 3 & $(9,76)$ & 0,07 & 0,04 & $=$ & 0 & 0 & - & - & - & - \\
\hline $\begin{array}{l}\text { Parapiptadenia } \\
\text { excelsa }\end{array}$ & 1 & 2 & $(4,88)$ & 0,05 & 0,01 & $=$ & 4 & 0 & - & - & - & - \\
\hline $\begin{array}{l}\text { Phyllostylon } \\
\text { rhamnoides }\end{array}$ & 38 & 2 & $(4,88)$ & 0,05 & 0,41 & -- & 0 & 0 & - & - & - & - \\
\hline $\begin{array}{l}\text { Anadenanthera } \\
\text { colubrina }\end{array}$ & 10 & 2 & $(4,88)$ & 0,05 & 0,08 & $=$ & 20 & 3 & $(6,67)$ & 0,07 & 0,13 & $=$ \\
\hline $\begin{array}{l}\text { Schinopsis } \\
\text { lorentzii }\end{array}$ & $<1$ & 2 & $(4,88)$ & 0,05 & 0,01 & $=$ & 7 & 4 & $(8,89)$ & 0,09 & 0,09 & $=$ \\
\hline $\begin{array}{l}\text { Aspidosperma } \\
\text { quebracho-blanco }\end{array}$ & 0 & 0 & - & - & - & - & 6 & 22 & $(48,89)$ & 0,49 & 0,14 & + \\
\hline $\begin{array}{l}\text { Chorisia } \\
\text { insignis }\end{array}$ & 3 & 0 & - & - & - & - & 1 & 13 & $(28,89)$ & 0,29 & 0,06 & + \\
\hline $\begin{array}{l}\text { Diatenopteryx } \\
\text { sorbifolia }\end{array}$ & 0 & 0 & - & - & - & - & 4 & 2 & $(4,44)$ & 0,04 & 0,04 & $=$ \\
\hline Árbol muerto & 12 & 4 & $(7,32)$ & 0,10 & 0,16 & $=$ & 7 & 1 & $(2,22)$ & 0,02 & 0,04 & $=$ \\
\hline
\end{tabular}

pueden haberse encontrado en árboles vivos dado que en estos bosques subtropicales los árboles muertos constituyen un porcentaje menor al $12 \%$ del total de los árboles en pie (Politi et al. 2010). El uso predominante de árboles vivos para excavar huecos también se registró para C. magellanicus (Ojeda et al. 2007), única especie del género con abundantes datos sobre huecos excavados, previo al presente trabajo. Campephilus imperialis y C. principalis, hoy probablemente extintos (Husak y Husak 2003), también son referidos como usuarios de árboles mayormente vivos para nidificar. Las demás especies del género son poco conocidas, pero parecerían excavar predominantemente en árboles muertos (C. melanoleucos (Kilham 1972), C. guatemaltensis (Kilham 1977) y C. pollens (Greeney et al. 2010) igual que lo reportado para otros géneros de pájaros carpinteros (Sandoval y Barrantes 2006). Para otros pícidos, sin embargo, los patrones de selección de sustratos vivos o muertos se desdibujan a medida que se las estudia en tipos forestales y condiciones diversas dentro de su rango de distribución, sugiriendo que el uso de un tipo u otro de sustrato dependería de las características forestales del sitio (Ojeda y Chazarreta 2014). Por lo tanto, se necesitan 
Cuadro 2. Media \pm desvío estándar del diámetro a la altura del pecho de las especies arbóreas con y sin hueco excavado por Campephilus leucopogon en la selva pedemontana de las Yungas Australes y en el bosque de dos quebrachos del Chaco Seco de la provincia de Jujuy, Argentina. Se indica la prueba U de Mann Whitney $(W)$ y el asterisco indica diferencias significativas $(P<0,05)$.

Mean and standard deviations of diameter at breast height of trees with and without excavated cavities by Campephilus leucopogon in Piedmont forest of the Southern Yungas and Dry Chaco forest in Jujuy Province, Argentina. U-test (W) and significant level $(P<0.05)$ are shown.

\begin{tabular}{|c|c|c|c|}
\hline Especie de árbol & Árboles con hueco & Árboles sin hueco & $\mathrm{W}$ \\
\hline \multicolumn{4}{|l|}{ Selva pedemontana } \\
\hline Calycophyllum multiflorum & $62,66 \pm 12,26$ & $32,25 \pm 17,20$ & $17^{*}$ \\
\hline Amburana cearensis & $68,85 \pm 16,55$ & $35,03 \pm 17,77$ & $100^{*}$ \\
\hline Astronium urundeuva & $81,31 \pm 25,60$ & $31,07 \pm 13,29$ & $784^{*}$ \\
\hline Cedrela balansae & $62,95 \pm 8,92$ & $22,38 \pm 7,25$ & $27^{*}$ \\
\hline Árbol muerto & $57,08 \pm 5,87$ & $24,51 \pm 13,63$ & $259^{*}$ \\
\hline Otros & $46,41 \pm 6,72$ & $23,89 \pm 12,84$ & $2013 *$ \\
\hline \multicolumn{4}{|l|}{ Bosque chaqueño } \\
\hline Aspidosperma quebracho-blanco & $56,31 \pm 9,24$ & $25,25 \pm 11,23$ & $207^{*}$ \\
\hline Chorisia insignis & $69,35 \pm 21,98$ & $41,06 \pm 3,22$ & $766^{*}$ \\
\hline Schinopsis lorentzii & $79,93 \pm 32,31$ & $31,78 \pm 22,55$ & $9 *$ \\
\hline Anadenanthera colubrina & $43,31 \pm 12,68$ & $17,65 \pm 5,89$ & $96^{*}$ \\
\hline Otros & $46,28 \pm 17,20$ & $15,55 \pm 12,31$ & $48^{*}$ \\
\hline
\end{tabular}

estudios de selección de sustratos en diferentes ambientes forestales ocupados por cada especie de pícido para lograr un entendimiento más integral de los patrones de uso de árboles para excavación. Para el carpintero lomo blanco, el uso mayoritario de árboles vivos en ambos tipos de bosque analizados, sugiere un patrón independiente del sitio, pero se debiera extender el estudio a otros sitios ocupados por la especie para poder confirmar esta generalización.

En ambos tipos de bosques, los huecos excavados por el carpintero lomo blanco se encontraron en árboles con un DAP similar $(60 \mathrm{~cm})$, aún cuando el DAP promedio en el bosque chaqueño de dos quebrachos fue significativamente menor que en la selva pedemontana. Este resultado podría sugerir que existe un tamaño mínimo de hueco necesario para que el carpintero lomo blanco pueda nidificar o refugiarse (Sandoval 2008). Además, se sugiere que la excavación de huecos en árboles de diámetro más chico es más susceptible a daños físicos, ya que las paredes del hueco serían de poco espesor (Sandoval 2008).

La diferencia encontrada en la altura de los huecos excavados por el carpintero lomo blanco en los distintos tipos de bosques (i.e., mayor altura en la selva pedemontana que en el bosque chaqueño de dos quebrachos) podría deberse a la diferencia en la altura del dosel de ambos tipos de bosques. En los huecos a mayor altura se registra mayor éxito reproductivo en especies de aves que nidifican en huecos ( $\mathrm{Li}$ y Martin 1991). Sería importante evaluar el éxito reproductivo del carpintero lomo blanco y comparar los valores entre estos dos tipos de bosques para corroborar esta hipótesis.
En ambos tipos de bosques estudiados, los huecos excavados por el carpintero lomo blanco se encontraron principalmente en troncos y ramas primarias que son las partes más antiguas y de mayor diámetro de los árboles. La ausencia de huecos excavados en ramas terciarias y cuaternarias en la selva pedemontana puede deberse a que estos son los sectores más susceptibles a romperse debido al viento o rayos (Jackson y Jackson 2004). En el bosque chaqueño de dos quebrachos la proporción de huecos excavados en troncos y ramas fue similar, lo cual puede relacionarse con que la dureza de la madera disminuye con la altura del árbol (Ćiković et al. 2014), sobre todo considerando que la mayoría de los huecos excavados se encontraron en Aspidosperma quebracho-blanco un árbol de madera considerada dura (Moglia et al. 2009).

La orientación de la entrada de los huecos excavados por el carpintero lomo blanco en ambos tipos de bosque tuvo una distribución uniforme. Se ha sugerido que la orientación de los huecos favorece las condiciones de microclimas dentro del hueco (Maziarz y Wesołowski 2013). Es probable que en estos bosques los huecos estén bien protegidos de la exposición al viento y humedad y no sea necesario seleccionar una orientación para mejorar las condiciones climáticas (Rico y Sandoval 2014).

Los resultados muestran que el carpintero lomo blanco selecciona ciertas especies arbóreas para excavar sus huecos (Calycophyllum multiflorum y Amburana cearensis en la selva pedemontana y Aspidosperma quebracho-blanco y Chorisia insignis en el bosque chaqueño de dos quebra- 
chos). Estas especies arbóreas son de valor maderable, salvo $C$. insignis, y son actualmente aprovechadas económicamente en forma intensiva (Giménez y Moglia 2003, Brown et al. 2009). Además, Amburana cearensis está clasificada como en peligro de extinción en la lista roja de la UICN. Calycophyllum multiflorum no solo es importante para el carpintero lomo blanco como sitios de excavación, sino que también es una especie arbórea que frecuentemente desarrolla huecos producto de la descomposición de la madera, que son utilizados para nidificar por varias especies de aves que nidifican en huecos (Politi et al. 2009). Esto resalta el valor clave de Calycophyllum multiflorum y obliga a generar recomendaciones de manejo para esta, y para las demás especies arbóreas seleccionadas por el carpintero lomo blanco para excavar huecos, para asegurar retener una adecuada representación de individuos de estas especies en sitios bajo aprovechamiento forestal. Además, la carencia de réplicas y un $n$ bajo de muestras, hacen que los resultados de este trabajo deban considerarse con precaución. Sin embargo, por ser este el primer trabajo con información recopilada sistemáticamente para el carpintero lomo blanco, puede resultar un aporte importante para la conservación de esta especie y de los bosques donde habita.

\section{CONCLUSIONES}

El carpintero lomo blanco selecciona sitios de excavación en árboles mayormente vivos, grandes (DAP > 60 $\mathrm{cm}$ ), de Calycophyllum multiflorum y Amburana cearensis en la selva pedemontana y de Aspidosperma quebrachoblanco y Chorisia insignis en el bosque chaqueño de dos quebrachos. Esta información sobre los requerimientos de hábitat para excavación de huecos por el carpintero lomo blanco puede contribuir a delinear pautas de manejo forestal sostenible que contemplen los requerimientos de hábitat del carpintero lomo blanco para excavar huecos. Asegurar el mantenimiento de poblaciones saludables de carpintero lomo blanco, al igual que otros pájaros carpinteros, puede ser determinante en la cantidad de huecos disponibles afectando directamente la diversidad y abundancia de especies no excavadoras.

\section{AGRADECIMIENTOS}

Agradecemos a Oscar Eduardo Vargas por su colaboración en la elaboración de los mapas, a Cecilia García por colaborar con los análisis estadísticos, a Miguel Ulivarri de la Empresa Ledesma S.A.A.I. por otorgarnos los permisos para realizar este trabajo. Dos revisores anónimos realizaron sugerencias que mejoraron sustancialmente el trabajo. Este trabajo se ha financiado con el aporte del FONCyT, a través del proyecto PICT-2012-0892 Préstamo BID, CONICET, a través del proyecto PIP $\mathrm{N}^{\circ} 259$, Idea Wild y RUFFORD. LR y NP son investigadores de CONICET; AS y CV son becarios doctorales de CONICET.

\section{REFERENCIAS}

Aitken KE, K Martin. 2007. The importance of excavators in hole-nesting communities: availability and use of natural tree holes in old mixed forests of western Canada. Journal of Ornithology 148(2): 425-434.

Brown AD, P Blendinger, T Lomáscolo, P García Bes. 2009. Selva Pedemontana de las Yungas. Historia natural, ecología y manejo de un ecosistema en peligro. San Miguel de Tucumán, Argentina. Ediciones del Subtrópico. 487 p.

Ćiković D, S Barišic, V Tutiš, J Kralj. 2014. Nest site and nesthole characteristics used by Great Spotted Woodpecker Dendrocopos major L. in Croatia. Polish Journal of Eco$\operatorname{logy}$ 62(2): 349-360.

Cornelius C, K Cockle, N Politi, I Berkunsky, L Sandoval, V Ojeda, L Rivera, M Hunter, K Martin. 2008. Cavity-nesting bird in Neotropical forest: cavities as a potentially limiting resource. Ornitologia Neotropical 19(suppl.): 253-268.

de la Peña MR. 2005. Reproducción de las aves argentinas (con descripción de pichones). Buenos Aires, Argentina. Editorial L.O.L.A. 845 p.

Fisher NI. 1993. Statistical analysis of circular data. Cambridge, Inglaterra. Cambridge University Press. 296 p.

Husak MS, AL Husak. 2003. Latitudinal patterns in range sizes of New World woodpeckers. The Southwestern Naturalist 48(1): 61-69.

Gasparri NI, HR Grau. 2009. Deforestation and fragmentation of Chaco dry forest in NW Argentina (1972-2007). Forest Ecology and Management 258(6): 913-921.

Giménez AM, JG Moglia. 2003. Árboles del Chaco Argentino. Guía para el Reconocimiento Dendrológico. Santiago del Estero, Argentina. Universidad de Santiago del Estero, Facultad de Ciencias Forestales. 307 p.

Greeney HF, J Simbaña, LA Salazar-V. 2010. First description of the eggs and nestlings of Powerful Woodpecker (Campephilus pollens). Boletín SAO 20: 5-11.

Jackson JA, BJ Jackson. 2004. Ecological relationships between fungi and woodpecker cavity sites. Condor 106(1): 37-49.

Kilham L. 1972. Habits of the Crimson-crested Woodpecker in Panama. The Wilson Bulletin 84: 28-47.

Kilham L. 1977. Nesting behavior of Pale-billed woodpeckers in Guatemala. Auk 94(4): 773-774.

Li P, TE Martin. 1991. Nest-site selection and nesting success of cavity-nesting birds in high elevation forest drainages. Auk 108(2): 405-418.

Manly BFJ, L McDonald, D Thomas. 1993. Resource selection by animals: statistical design and analysis for field studies. Londres, Reino Unido. Chapman and Hall. 219 p.

Maziarz M, T Wesołowski. 2013. Microclimate of tree cavities used by Graet Tits (Parus major) in a primaveral forest. Avian Biology Research 6(1): 47-56.

Moglia, JG, S Bravo, AM Giménez, C López. 2009. ¿Son los caracteres estructurales de la madera de Aspidosperma quebracho-blanco Schelkt causantes de su inestabilidad? Quebracho 17(1,2): 58-63.

Ojeda V, L Chazarreta. 2014. Home range and habitat use by Magellanic woodpeckers in old-growth forest of Patagonia. Canadian Journal of Forest Research 44(10): 1265-1273.

Ojeda V, L Suarez, T Kitzberger. 2007. Crown dieback events as key processes creating cavity habitat for Magellanic Woodpeckers. Austral Ecology 32 (4): 436-445. 
Politi N, MJ Hunter, L Rivera. 2009. Nest Selection by Cavitynesting Birds in Subtropical Montane Forests of the Andes: Implications for Sustainable Forest Management. Biotropica 41(3): 354-360.

Politi N, MJ Hunter, L Rivera. 2010. Availability of cavities for avian cavity nesters in selectively logged subtropical montane forests of the Andes. Forest Ecology and Management 260(5): 893-906.

Politi N, MJ Hunter, L Rivera. 2012. Assessing the effects of selective logging on birds in Neotropical piedmont and cloud montane forests. Biodiversity and Conservation 21(12):
3131-3155.

Rico D, L Sandoval. 2014. Non random orientation in woodpecker cavity in a tropical rain forest. Ornitología Neotropical 25: $1-10$.

Sandoval L. 2008. Implications of nest-site size selection on the nest building strategy in woodpeckers (Picidae: Aves). Métodos en Ecología y Sistemática 3(2): 1-5.

Winkler H, DA Christie. 2002. Family Picidae (woodpeckers). In del Hoyo J, A Elliot, J Sargatal eds. Handbook of the birds of the world. Volume 7. Barcelona, España. Lynx Edicions. p. 296-558. 